\title{
Kernels, truth and satisfaction
}

by

\author{
James H. SCHMERL \\ Presented by Feliks PRZYTYCKI
}

Summary. The Kotlarski-Krajewski-Lachlan Theorem says that every resplendent model of Peano Arithmetic has a full satisfaction class. Enayat and Visser gave a more modeltheoretic proof of this theorem. We redo their proof using kernels of directed graphs.

Prologue. A fundamental theorem of Tarski asserts that truth in arithmetic is not arithmetically definable. Nevertheless, there is the well-known Kotlarski-Krajewski-Lachlan Theorem [KKL81] that says that every model $\mathcal{M}$ of Peano Arithmetic (PA) has an elementary extension $\mathcal{N} \succ \mathcal{M}$ having a full satisfaction class (and even a full truth class). Roughly, a full truth class is a subset of the model that satisfies the usual recursive definition of truth: the atomic sentences in the set are precisely the true ones; a disjunction $\sigma_{1} \vee \sigma_{2}$ is in the set iff at least one of $\sigma_{1}, \sigma_{2}$ is in the set; an existentially quantified sentence $\exists x \varphi(x)$ is in the set iff some instantiation $\varphi(c)$ of it is in the set; etc. Over 30 years later, Enayat \& Visser [EV15] gave another proof of the KKL Theorem. According to [EV15], the proof in [KKL81] used some "rather exotic proof-theoretic technology", while the proof in [EV15] uses "a perspicuous method for the construction of full satisfaction classes". Although not made explicit there, the proof in [EV15], when stripped to its essentials, is seen to ultimately depend on showing that certain digraphs have kernels. This is made explicit here.

There is a lengthy discussion in [EV15, §4] about the relationship of full satisfaction classes to full truth classes. Satisfaction classes, which are sets of ordered pairs consisting of a formula in the language of arithmetic and an

2010 Mathematics Subject Classification: Primary 03H15, 03C50, 05C20.

Key words and phrases: satisfaction classes, Peano Arithmetic, directed graphs, kernels. Received 24 November 2018.

Published online 1 March 2019. 
assignment for that formula, are exclusively used in [EV15]. Truth classes are sets of arithmetic sentences that may also have domain constants. By EV15, Prop. 4.3] (whose "routine but laborious proof is left to the reader"), there is a canonical correspondence between full truth classes and extensional full satisfaction classes. The culmination of [EV15, §4] is the construction of extensional full satisfaction classes. In $\S 2$ of this paper, we will avoid the intricacies of [EV15, §4] by working exclusively with truth classes to easily obtain the same conclusion.

1. Digraphs and kernels. A binary relational structure $\mathcal{A}=(A, E)$ is referred to here as a directed graph, or digraph for short $\left(^{1}\right)$. A subset $K \subseteq A$ is a kernel of $\mathcal{A}$ if for every $a \in A, a \in K$ iff whenever $a E b$, then $b \notin K$. According to BJG09, kernels were introduced by von Neumann vNM44 and have subsequently found many applications. For $n<\omega$, define the binary relation $E^{n}$ on $A$ by recursion: $x E^{0} y$ iff $x=y ; x E^{n+1} y$ iff $x E z$ and $z E^{n} y$ for some $z \in A$. A digraph $\mathcal{A}$ is a directed acyclic graph $(D A G)$ if whenever $n<\omega$ and $a E^{n} a$, then $n=0$. Some DAGs have kernels while others do not. For example, if $<$ is a linear order of $A$ with no maximum element, then $(A,<)$ is a DAG with no kernel. However, every finite DAG has a (unique) kernel, as was first noted in vNM44.

An element $b \in A$ for which there is no $c \in A$ such that $b E c$ is a sink of $\mathcal{A}$. We say that $\mathcal{A}$ is well-founded if every nonempty subdigraph of $\mathcal{A}$ has a sink. Every finite DAG is well-founded, and every well-founded digraph is a DAG having a kernel. The next proposition, for which we need some more definitions, says even more is true. A subset $D$ of a digraph $\mathcal{A}$ is closed if whenever $d \in D$ and $d E a$, then $a \in D$. If $X \subseteq A$ and $k<\omega$, then define $\mathrm{Cl}_{k}^{\mathcal{A}}(X)$ by recursion: $\mathrm{Cl}_{0}^{\mathcal{A}}(X)=X$ and $\mathrm{Cl}_{k+1}^{\mathcal{A}}(X)=X \cup\{a \in A$ : $d E a$ for some $\left.d \in \mathrm{Cl}_{k}^{\mathcal{A}}(X)\right\}$. Let $\mathrm{Cl}^{\mathcal{A}}(X)=\bigcup_{k<\omega} \mathrm{Cl}_{k}^{\mathcal{A}}(X)$, which is the smallest closed superset of $X$.

Proposition 1. Suppose that $\mathcal{A}$ is a digraph, $D \subseteq A$ is closed, $K_{0} \subseteq D$ is a kernel of $D$, and $A \backslash D$ is well-founded. Then $\mathcal{A}$ has a (unique) kernel $K$ such that $K_{0}=K \cap D$.

Proof. By recursion on ordinals $\alpha$, define $D_{\alpha}$ so that $D_{0}=D, D_{\alpha+1}=$ $D_{\alpha} \cup\left\{b \in A: b\right.$ is a sink of $\left.A \backslash D_{\alpha}\right\}$, and $D_{\alpha}=\bigcup_{\beta<\alpha} D_{\beta}$ if $\alpha$ is a limit ordinal. Let $\gamma$ be such that $A=D_{\gamma}$. Every $D_{\alpha}$ is closed. Define $K_{\alpha}$ as follows: if $\alpha=\beta+1$, then $K_{\alpha}=K_{\beta} \cup\left\{a \in D_{\alpha} \backslash D_{\beta}: a E b\right.$ for no $\left.b \in K_{\beta}\right\}$; if $\alpha$ is a limit ordinal, then $K_{\alpha}=\bigcup\left\{K_{\beta}: \beta<\alpha\right\}$. Let $K=K_{\gamma}$. One easily proves, by induction on $\alpha$, that $K_{\alpha}$ is the unique kernel of $D_{\alpha}$ such that $K_{0}=K_{\alpha} \cap D_{\alpha}$. Let $K=K_{\gamma}$.

$\left({ }^{1}\right)$ Henceforth, $\mathcal{A}$ always denotes a digraph $(A, E)$. If $B \subseteq A$, then we often identify $B$ with the the induced subdigraph $\mathcal{B}=\left(B, E \cap B^{2}\right)$. 
Let $\mathcal{A}$ be a digraph. If there is $k<\omega$ for which there are no $a, b \in A$ such that $a E^{k+1} b$, then $\mathcal{A}$ has finite height, and we let $\operatorname{ht}(\mathcal{A})$, the height of $\mathcal{A}$, be the least such $k$. If $\mathcal{A}$ has finite height, then it is well-founded. We say that $\mathcal{A}$ has local finite height if for every $m<\omega$ there is $k<\omega$ such that ht $\left(\mathrm{Cl}_{m}^{\mathcal{A}}(F)\right) \leq k$ for every $F \subseteq A$ having cardinality at most $m$. If $\mathcal{A}$ has local finite height, then it is a DAG. Having local finite height is a first-order property: if $\mathcal{B} \equiv \mathcal{A}$ and $\mathcal{A}$ has local finite height, then so does $\mathcal{B}$.

THEOREM 2. Every digraph $\mathcal{A}$ having local finite height has an elementary extension $\mathcal{B} \succ \mathcal{A}$ that has a kernel.

Proof. This proof is modeled after Theorem 3.2(b)'s in EV15.

To get $\mathcal{B}$ with a kernel $K$, we let $B_{0}=\emptyset$, and then obtain an elementary chain $\mathcal{A}=\mathcal{B}_{1} \prec \mathcal{B}_{2} \prec \cdots$ and an increasing sequence $\emptyset=K_{0} \subseteq K_{1} \subseteq$ $K_{2} \subseteq \cdots$ such that for every $n<\omega, K_{n}$ is a kernel of $\mathrm{Cl}^{\mathcal{B}_{n+1}}\left(B_{n}\right)$ and $K_{n}=K_{n+1} \cap B_{n}$. Having these sequences, we let $\mathcal{B}=\bigcup_{n<\omega} \mathcal{B}_{n+1}$ and $K=\bigcup_{n<\omega} K_{n}$. Clearly, $\mathcal{B} \succ \mathcal{A}$ and $K$ is a kernel of $\mathcal{B}$.

We construct these sequences by recursion. Notice that we have $\mathcal{B}_{0}, \mathcal{B}_{1}$ and $K_{0}$ at the start. Now suppose that we have $B_{n}, \mathcal{B}_{n+1}$ and $K_{n}$ such that $\mathcal{A} \preccurlyeq \mathcal{B}_{n+1}, B_{n}$ is a closed subset of $B_{n+1}$ and $K_{n}$ is a kernel of $D=$ $\mathrm{Cl}^{\mathcal{B}_{n+1}}\left(B_{n}\right)$. We obtain $\mathcal{B}_{n+2}$ and $K_{n+1}$.

Let $\Sigma$ be the union of the following three sets of sentences:

- $\operatorname{Th}\left(\left(\mathcal{B}_{n+1}, a\right)_{a \in B_{n+1}}\right)$;

- $\left\{\sigma_{F, k}: k<\omega\right.$ and $F \subseteq B_{n+1}$ is finite $\}$, where $\sigma_{F, k}$ is the sentence

$$
\forall x \in \mathrm{Cl}_{k}(F)\left[U(x) \leftrightarrow \forall y \in \mathrm{Cl}_{k+1}(F)(x E y \rightarrow \neg U(y))\right] ;
$$

- $\left\{U(d): d \in K_{n}\right\} \cup\left\{\neg U(d): d \in D \backslash K_{n}\right\}$.

This $\Sigma$ is a set of $\mathcal{L}$-sentences, where $\mathcal{L}=\{E, U\} \cup B_{n+1}$ and $U$ is a new unary relation symbol.

It suffices to show that $\Sigma$ is consistent, for then we can let $\left(\mathcal{B}_{n+2}, U\right) \models \Sigma$ and let $K_{n+1}=U \cap \mathrm{Cl}^{\mathcal{B}_{n+2}}\left(B_{n+1}\right)$. To do so, we need only show that every finite subset of $\Sigma$ is consistent.

Let $\Sigma_{0} \subseteq \Sigma$ be finite. Let $k_{0}<\omega$ and finite $F_{0} \subseteq B_{n+1}$ be such that if $\sigma_{F, k} \in \Sigma_{0}$, then $k<k_{0}$ and $F \subseteq F_{0}$. Let $D_{0}=\mathrm{Cl}_{k_{0}}^{\mathcal{B}_{n+1}}\left(F_{0}\right)$ and let $D_{1}=D_{0} \cup D$. Since $\mathcal{B}_{n+1}$ has local finite height, $D_{0}$ has finite height, and therefore is well-founded, so $D_{1} \backslash D$ is well-founded. Since $D$ is a closed subset of $B_{n+1}$, it is also a closed subset of $D_{1}$. We can now apply Proposition 1 to get a kernel $U$ of $D_{1}$ such that $K_{n}=U \cap D$. Then $\left(\mathcal{B}_{n+1}, U\right) \models \Sigma_{0}$, so $\Sigma_{0}$ is consistent.

Thus, Theorem 2 is proved.

Recall that $\mathcal{A}$ is resplendent iff whenever $\sigma$ is a first-order $(\{E, R\} \cup A)$ sentence, where $R$ is some new $k$-ary relation symbol, such that $(\mathcal{B}, S) \models \sigma$ 
for some $\mathcal{B} \succ \mathcal{A}$ and $S \subseteq B^{k}$, then there already is $R \subseteq A^{k}$ such that $(\mathcal{A}, R) \models \sigma$. Every $\mathcal{A}$ has a resplendent elementary extension of the same cardinality. In general, resplendent digraphs are recursively saturated, and conversely, all countable, recursively saturated digraphs are resplendent [BS76].

COROLlary 3. Every resplendent (or countable, recursively saturated) digraph that has local finite height has a kernel.

2. Truth classes. There are various ways that syntax for arithmetic can be defined in a model $\mathcal{M}$ of PA. It usually makes little difference how it is done, so we will choose a way that is very convenient.

We will formalize the language of arithmetic by using just two ternary relation symbols: one for addition and one for multiplication. Suppose that $\mathcal{M} \models$ PA. For each $a \in M$, we have a constant symbol $c_{a}$. Let $\mathcal{L}^{M}$ consist of the two ternary relations and all the $c_{a}$ 's. The only propositional connective we will use is the NOR connective $\downarrow$, where $\sigma_{0} \downarrow \sigma_{1}$ is $\neg\left(\sigma_{0} \vee \sigma_{1}\right)$. The only quantifier we will use is the "there are none such that" quantifier $\boldsymbol{U}$, where И $v \varphi(v)$ is $\forall v[\neg \varphi(v)]$. Notice that the usual connectives and quantifiers can be defined in terms of these new ones; for example, let $\neg \sigma=\sigma \downarrow \sigma, \sigma_{1} \vee \sigma_{2}=$ $\neg\left(\sigma_{1} \downarrow \sigma_{2}\right)$ and $\exists v \varphi(v)=\neg И v \varphi(v)$. Let Sent ${ }^{\mathcal{M}}$ be the set of $\mathcal{L}^{\mathcal{M}}$-sentences as defined in $\mathcal{M}$. A subset $S \subseteq$ Sent $^{\mathcal{M}}$ is a full truth class for $\mathcal{M}$ provided the following hold for every $\sigma \in$ Sent $^{\mathcal{M}}$ :

- if $\sigma=\sigma_{0} \downarrow \sigma_{1}$, then $\sigma \in S$ iff $\sigma_{0}, \sigma_{1} \notin S$;

- if $\sigma=\operatorname{\eta n} v(v)$, then $\sigma \in S$ iff there is no $a \in M$ such that $\varphi\left(c_{a}\right) \in S$;

- if $\sigma$ is atomic, then $\sigma \in S$ iff $\mathcal{M} \models \sigma$.

Notice that a full truth set behaves properly when restricted to the usual connectives and quantifiers.

Let $A^{\mathcal{M}}=\left\{\sigma \in\right.$ Sent $^{\mathcal{M}}$ : if $\sigma$ is atomic, then $\left.\mathcal{M}=\sigma\right\}$. Define the binary relation $E^{\mathcal{M}}$ on $A^{\mathcal{M}}$ so that if $\sigma_{1}, \sigma_{2} \in A^{\mathcal{M}}$, then $\sigma_{2} E^{\mathcal{M}} \sigma_{1}$ iff one of the following holds:

- there is $\sigma_{0}$ such that $\sigma_{2}=\sigma_{0} \downarrow \sigma_{1}$ or $\sigma_{2}=\sigma_{1} \downarrow \sigma_{0}$;

- $\sigma_{2}=\operatorname{hv} \varphi(v)$ and $\sigma_{1}=\varphi\left(c_{a}\right)$ for some $a \in M$.

Consider the digraph $\mathcal{A}=\mathcal{A}^{\mathcal{M}}=\left(A^{\mathcal{M}}, E^{\mathcal{M}}\right)$. We easily see that $S$ is a full truth class for $\mathcal{M}$ iff $S$ is a kernel of $\mathcal{A}$.

Obviously, $\mathcal{A}$ is a DAG. Moreover, it has local finite height: if $F \subseteq A^{\mathcal{M}}$ is finite and $m<\omega$, then $\operatorname{ht}\left(\mathrm{Cl}_{m}^{\mathcal{A}}(F)\right) \leq\left(2^{m+1}-1\right)|F|$. (A hint for proving this: Define the equivalence relation $\sim$ on Sent ${ }^{\mathcal{M}}$ so that $\sigma_{1} \sim \sigma_{2}$ iff $\sigma_{1}^{\prime}=\sigma_{2}^{\prime}$, where $\sigma_{e}^{\prime}$ results after replacing all constant symbols in $\sigma_{e}$ with $c_{0}$. If $\sigma_{0} \sim \tau_{0}$ and $\sigma_{0}, \tau_{0} \in A^{\mathcal{M}}$, then there are $\sigma_{1}, \sigma_{2} \in \mathrm{Cl}_{1}^{\mathcal{A}}\left(\left\{\sigma_{0}\right\}\right)$ such that for every $\tau \in \operatorname{Cl}_{1}^{\mathcal{A}}\left(\left\{\tau_{0}\right\}\right)$ there is $e \leq 2$ such that $\tau \sim \sigma_{e}$.) We can now infer the following version of the KKL Theorem. The comments preceding Corollary 3 
about resplendent digraphs apply mutatis mutandis to resplendent models of PA.

COROLLARY 4. Every resplendent (or countable, recursively saturated) $\mathcal{M}=$ PA has a full truth class.

Proof. Since $\mathcal{A}^{\mathcal{M}}$ is definable in $\mathcal{M}$ and $\mathcal{M}$ is resplendent, $\mathcal{A}^{\mathcal{M}}$ is also resplendent. Thus, by Corollary $3, \mathcal{A}^{\mathcal{M}}$ has a kernel, which we have seen is a full truth class for $\mathcal{M}$.

Corollary 4 can be improved by replacing PA with any of its subtheories in which enough syntax is definable. Also, Corollary 4 can be applied to expansions of models of PA. To see an example, let Const $^{\mathcal{M}}$ be the set of constant $\mathcal{L}^{\mathcal{M}}$-terms as defined in $\mathcal{M}$ (in which + and $\times$ are considered as function symbols). Let $\mathcal{M}$ be resplendent, and let $I$ be the definable binary relation on Const $^{M}$ such that for any $s, t \in$ Const $^{\mathcal{M}},\langle s, t\rangle \in I$ iff $s^{\mathcal{M}}=t^{\mathcal{M}}$. By applying Corollary 4 to $(\mathcal{M}, I)$, we conclude that $\mathcal{M}$ has a full truth class $S$ such that for all $s, t \in$ Const $^{\mathcal{M}}$, the sentence $s=t$ is in $S$ iff $s^{\mathcal{M}}=t^{\mathcal{M}}$.

\section{References}

[BJG09] J. Bang-Jensen and G. Gutin, Digraphs. Theory, Algorithms and Applications, 2nd ed., Springer Monogr. Math., Springer, London, 2009.

[BS76] J. Barwise and J. Schlipf, An introduction to recursively saturated and resplendent models, J. Symbolic Logic 41 (1976), 531-536.

[EV15] A. Enayat and A. Visser, New constructions of satisfaction classes, in: Unifying the Philosophy of Truth, Log. Epistemol. Unity Sci. 36, Springer, Dordrecht, 2015, 321-335.

[KKL81] H. Kotlarski, S. Krajewski and A. H. Lachlan, Construction of satisfaction classes for nonstandard models, Canad. Math. Bull. 24 (1981), 283-293.

[vNM44] J. von Neumann and O. Morgenstern, Theory of Games and Economic Behavior, Princeton Univ. Press, Princeton, 1944.

James H. Schmerl

Department of Mathematics

University of Connecticut

Storrs, CT 06269, U.S.A.

E-mail: james.schmerl@uconn.edu 
